\title{
Avaliação dos compostos bioativos e capacidade antioxidante em cultivares de feijão- caupi (Vigna unguiculata L.) imaturo cru, cozido e seus caldos de cocção
}

\author{
Evaluation of bioactive compounds and antioxidant capacity in raw, cooked immature cowpea
}

(Vigna unguiculata L.) cultivars and their cooking broths

Evaluación de compuestos bioactivos y capacidad antioxidante en cultivares de caupi inmaduro crudo cocido (Vigna unguiculata L.) y sus caldos de cocción

\author{
Maria Marlene da Silva Vieira \\ ORCID: https://orcid.org/0000-0002-2085-7461 \\ Universidade Federal de Campina Grande, Brasil \\ E-mail: mmdsv2708@hotmail.com \\ Júlia Medeiros Bezerra \\ ORCID: https://orcid.org/0000-0001-7662-2488 \\ Universidade Federal de Campina Grande, Brasil \\ E-mail: juliamedeiros1709@hotmail.com \\ Adriana Ferreira dos Santos \\ ORCID: https://orcid.org/0000-0002-9356-8054 \\ Universidade Federal da Paraíba, Brasil \\ E-mail: adrefesantos@yahoo.com
}

\begin{abstract}
Resumo
O feijão é um dos alimentos mais produzidos em todo o território nacional, sendo intensa a busca por cultivares produtivas e com boas características culinárias. Tendo em vista a preocupação crescente dos consumidores com a dieta, as positivas implicações de alimentos funcionais na saúde e os benefícios do feijão para o organismo, objetivouse nesse estudo verificar o conteúdo de compostos bioativos e a capacidade antioxidante de oito cultivares de feijãocaupi, antes e após o cozimento, assim como em seus respectivos caldos. Das amostras foram determinadas as concentrações de ácido ascórbico, clorofila, carotenoides, antocianinas, flavonoides, compostos fenólicos e capacidade antioxidante, e realizada a comparação das médias dos dados obtidos, pelo teste de Tukey ao nível de 5\% de probabilidade. Como resultados, observou-se que a cultivar Costela de Vaca apresentou as maiores concentrações de clorofila e carotenoides antes e após o cozimento. Houve um aumento na concentração de flavonoides e redução do teor de ácido ascórbico após o cozimento em todas as cultivares avaliadas. A cultivar BRS Marataoã apresentou a maior concentração de compostos fenólicos no grão cru e consequentemente maior capacidade antioxidante, porem com redução da atividade após o cozimento. Para os caldos de cocção, a BRS Marataoã e Costela de Vaca foram as que apresentaram maior capacidade em combater radicais livres. Apesar de o cozimento ter provocado alterações nas concentrações dos compostos bioativos, as cultivares mantiveram-se com características funcionais importantes, recomendando-se o consumo do feijão-caupi verde com o caldo de cocção para uma maior retenção de compostos com propriedades antioxidantes.
\end{abstract}

Palavras-chave: Feijão verde; Cozimento; Compostos fenólicos; Antioxidantes; Qualidade.

\begin{abstract}
Beans are one of the most produced foods in the entire national territory, with an intense search for productive cultivars with good culinary characteristics. In view of the growing concern of consumers with the diet, the positive implications of functional foods for health and the benefits of beans for the organism, the objective of this study was to verify the content of bioactive compounds and the antioxidant capacity of eight common bean cultivars. cowpea, before and after cooking, as well as in their respective broths. From the samples, the concentrations of ascorbic acid, chlorophyll, carotenoids, anthocyanins, flavonoids, phenolic compounds and antioxidant capacity were determined, and the data obtained were compared using the Tukey test at the 5\% probability level. As a result, it was observed that the cultivar Costela de Vaca had the highest concentrations of chlorophyll and carotenoids before and after cooking. There was an increase in the concentration of flavonoids and a reduction in the ascorbic acid content after cooking in all evaluated cultivars. The cultivar BRS Marataoã showed the highest concentration of phenolic compounds in the raw grain and, consequently, the highest antioxidant capacity, however with reduced activity after cooking. For cooking broths, BRS Marataoã and Costela de Vaca were the ones that had the greatest capacity to fight free radicals. Although cooking caused changes in the concentrations of bioactive compounds, the cultivars remained with
\end{abstract}


important functional characteristics, recommending the consumption of green cowpea with cooking broth for greater retention of compounds with antioxidant properties.

Keywords: Green beans; Cooking; Phenolic compounds; Antioxidants; Quality.

\section{Resumen}

El frijoles uno de los alimentos más producidos en todo el territorio nacional, con una intensa búsqueda de cultivares productivos con buenas características culinarias. Ante la creciente preocupación de los consumidores por la dieta, las implicaciones positivas de los alimentos funcionales para la salud y los beneficios del frijol para el organismo, el objetivo de este estudio fue verificar el contenido de compuestos bioactivos y la capacidad antioxidante de ocho frijoles comunes. cultivares de caupí, antes y después de la cocción, así como en sus respectivos caldos. A partir de las muestras se determinaron las concentraciones de ácido ascórbico, clorofila, carotenoides, antocianinas, flavonoides, compuestos fenólicos y capacidad antioxidante, y se compararon los promedios de los datos obtenidos mediante la prueba de Tukey al nivel de 5\% de probabilidad. Como resultado, se observó que el cultivar Costela de Vaca presentó las mayores concentraciones de clorofila y carotenoides antes y después de la cocción. Hubo un aumento en la concentración de flavonoides y una reducción en el contenido de ácido ascórbico después de la cocción en todos los cultivares evaluados. El cultivar BRS Marataoã mostró la mayor concentración de compuestos fenólicos en el grano crudo y, en consecuencia, la mayor capacidad antioxidante, sin embargo con una actividad reducida después de la cocción. Para los caldos de cocción, BRS Marataoã y Costela de Vaca fueron los que tuvieron mayor capacidad para combatir los radicales libres. Si bien la cocción provocó cambios en las concentraciones de compuestos bioactivos, los cultivares se mantuvieron con características funcionales importantes, recomendando el consumo de caupí verde con caldo de cocción para una mayor retención de compuestos con propiedades antioxidantes.

Palabras clave: Judias verdes; Cocción; Compuestos fenólicos; Antioxidantes; Calidad.

\section{Introdução}

O feijão-caupi (Vigna unguiculata (L.) Walp.) é uma das leguminosas mais plantadas e consumidas nas regiões Norte e Nordeste do Brasil, visto que suas sementes e vagens são utilizadas tanto para o consumo humano como importante fonte de proteína, energia, fibras e minerais, quanto como matéria-prima para adubação verde e material orgânico, sendo conhecida por diversas denominações regionais como: feijão-macassar, feijão-de-catador e feijão-de-corda, no Nordeste; feijão-de-praia, feijão-de-rama e feijão-de-estrada, na região Norte; feijão-miúdo, na região Sul e feijão-fradinho, no estado do Rio de Janeiro (Manaf; Zayed, 2015; Pereira et al., 2016).

Explorado tradicionalmente nos sistemas agrícolas familiares, em cultivo de sequeiro e com baixo nível tecnológico, o feijão-caupi cultivado nessas regiões ainda possui baixa produtividade de grãos, porém, tem sido pesquisado mais intensamente nas últimas décadas. Fato que tem contribuído para melhorar sua produtividade e rentabilidade, onde, associado a outros fatores, vêm despertando o interesse de médios e grandes produtores pela cultura. A adoção de tecnologias mais avançadas, como o uso de cultivares melhoradas, resistentes às doenças e com tolerância à seca, que em conjunto com um sistema de irrigação eficiente vem possibilitando a obtenção de altas produtividades, tanto para agricultores familiares quanto para empresariais, garantindo emprego, renda e alimento à população (Silva Junior, 2018; Locatelli, et al., 2014).

O feijão-caupi apresenta uma grande variabilidade genética que o torna versátil e é usado para várias finalidades e em diversos sistemas de produção. No Brasil, há três segmentos de mercado para essa cultura: grãos secos, grãos imaturos (feijão fresco ou verde) e sementes, sendo esses dois últimos de mercados crescentes. O termo "verde" se refere mais ao estádio em que a vagem é colhida do que propriamente à cor dos grãos, ou seja, corresponde à fase em que as vagens estão próximas da maturação, quando elas param de acumular fotossintatos, e os grãos apresentam umidade em torno de 60 a $70 \%$. Muito apreciado pelo seu sabor e fácil preparo, os grãos imaturos são usados em vários pratos típicos do Nordeste, sendo o chamado 'baião-de-dois' o mais popular, onde feijão-caupi e arroz são cozidos juntos (Andrade et al., 2011). É um produto com grande potencial para a expansão do consumo, como também para o processamento industrial nas formas de enlatamento, resfriamento e congelamento (Freire Filho, 2011; Silva et al., 2013).

A composição química e nutricional do feijão-caupi varia consideravelmente conforme a cultivar, seus grãos possuem compostos bioativos com efeitos benéficos à saúde humana (Sombié et al., 2018). Dentre estes compostos, destacam-se os 
polifenóis, que apresentam elevada atividade antioxidante e concentram-se especialmente no tegumento, conferindo a maior parte da coloração das sementes (Barros et al., 2017; Nderitu et al., 2013). As principais categorias de polifenóis presentes nas sementes de leguminosas, particularmente no caupi, são os ácidos fenólicos e flavonoides. Além disso, a quantidade de compostos fenólicos e as propriedades funcionais variam conforme a cultivar em estudo, e o processamento térmico aplicado (Cavalcante et al., 2017). Para agregar valor a essa cultura e evidenciar suas características funcionais, a identificação de compostos antioxidantes é de suma importância para o aumento de seu consumo (Moreira-Araújo et al., 2018).

Dessa forma, devido ao pouco conhecimento sobre os aspectos funcionais dessa leguminosa, o presente estudo foi realizado com o objetivo de determinar a presença de compostos bioativos e antioxidantes em diferentes cultivares de feijãocaupi imaturo, por este ser um alimento de fácil acesso, regional e fazer parte do hábito alimentar de grande parte da população brasileira.

\section{Metodologia}

O estudo experimental foi conduzido no Laboratório de Tecnologia de Produtos de Origem Vegetal (LTPOV) no Centro de Ciências e Tecnologia Agroalimentar, da Universidade Federal de Campina Grande, Pombal/PB. Foram analisadas oito cultivares de feijão-caupi imaturo. Costela de Vaca, BRS Marataoã, BRS Itaim, BR 17-Gurgueia, BRS Novaera, Paulistinha, Setentão e Patativa, fornecidas pelo setor de agricultura da Unidade Acadêmica de Ciências Agrárias do Centro de Ciências e Tecnologia Agroalimentar, da Universidade Federal de Campina Grande, Pombal/PB. As vargens imaturas foram debulhadas e os grãos separados em dois grupos: uma parte dos grãos crus foi macerada em almofariz com auxílio de um pistilo até obtenção de uma massa homogênea, enquanto outra parte foi submetida à cocção em panela de pressão doméstica em uma proporção feijão: água de 1:3 (p/v) durante 10 minutos. As amostras de feijão-cru, cozido e caldo foram armazenadas em potes plásticos $\left(-18^{\circ} \mathrm{C}\right)$ até o momento das análises dos compostos bioativos e atividade antioxidante, não sendo realizada a determinação de clorofila e carotenoides dos caldos de cocção.

\section{Delineamento Experimental e Análise Estatística}

O experimento foi instalado em um delineamento inteiramente casualizado e os resultados submetidos à análise de variância $(\mathrm{p}<0,05)$. Os tratamentos foram representados pelas cultivares (8) e as repetições (4) foram de $150 \mathrm{~g}$ de feijão e $50 \mathrm{ml}$ de caldo dos feijões-crus e cozidos, os quais foram avaliados independentemente. Os resultados obtidos foram submetidos à análise de variância e verificando efeito significativo para o teste F, os dados foram submetidos ao teste de Tukey ao nível de 5\% de probabilidade, utilizando o programa computacional ASSISTAT® 2016.

\section{Avaliação dos Compostos Bioativos e da Capacidade Antioxidante}

O teor de ácido ascórbico $\left(\mathrm{mg} .100^{-1} \mathrm{~g}^{-1}\right)$ foi determinado, segundo AOAC (2005), através da titulação com 2,6 diclorofenolindofenol (DFI), até obtenção de coloração rósea claro permanente, utilizando-se $1 \mathrm{~g}$ da amostra diluída em $50 \mathrm{~mL}$ de ácido oxálico (0,5\%).

As concentrações de clorofila (mg.100-1 $\left.\mathrm{g}^{-1}\right)$ e carotenoides cotais $\left(\mu \mathrm{g} \cdot 100^{-1} \mathrm{~g}^{-1}\right)$ foram determinadas de acordo com Lichtenthaler (1987), onde as amostras foram maceradas com acetona (80\%) gelada e carbonato de cálcio $\left(\mathrm{CaCO}_{3}\right)$, sendo centrifugadas e os sobrenadantes lidos em espectrofotômetro nos comprimentos de onda de 470, 646 e $663 \mathrm{~nm}$.

Os flavonoides e antocianinas (mg. $\left.100^{-1} \mathrm{~g}^{-1}\right)$ foram determinados conforme metodologia de Francis (1982), com as amostras maceradas em etanol- $\mathrm{HCl}(1,5 \mathrm{~N})$ na proporção 85:15 em ambiente escuro e deixados em repouso por 24 horas na geladeira. As amostras foram então filtradas e as leituras realizadas em espectrofotômetro a 374 e $535 \mathrm{~nm}$ para flavonoides e antocianinas respectivamente. 
Para a quantificação dos Polifenóis Extraíveis Totais - PET (mg.100-1 $\mathrm{g}^{-1}$ de ácido gálico) foi seguido método de FolinCiocalteau descrito por Waterhouse (2011), sendo a curva padrão preparada com ácido gálico e as leituras das amostras realizadas em espectrofotômetro a $765 \mathrm{~nm}$.

Para a avaliação da capacidade antioxidante, foi utilizado o método sequestrante do radical livre DPPH (1,1-difenil-2picrilidrazil), a começar pela obtenção do extrato alcoólico (metanol/acetona) do feijão que a partir do qual foram utilizadas alíquotas em diferentes concentrações com a solução de DPPH. As leituras foram realizadas a $515 \mathrm{~nm}$, observando-se a redução da absorbância até sua estabilização. O resultado foi expresso na forma de $\mathrm{EC}_{50}$ (g de feijão/g de DPPH), que corresponde à concentração da amostra necessária para reduzir em 50\% a concentração inicial do radical DPPH (Rufino et al., 2007).

\section{Resultados e Discussão}

No Quadro 1 encontram-se descritos os resultados referentes às médias dos valores de ácido ascórbico das oito cultivares de feijão-caupi e seus respectivos caldos. Observa-se que o teor de ácido ascórbico entre os grãos crus variou de 4,15 $\mathrm{mg} / 100 \mathrm{~g}$ (BRS Itaim) a 8,29 mg/100g (Costela de Vaca) e após o cozimento houve uma redução da concentração na maior parte as cultivares, com exceção da BRS Itaim $(4,23 \mathrm{mg} / 100 \mathrm{~g})$ e da Patativa $(5,56 \mathrm{mg} / 100 \mathrm{~g})$, as quais apresentaram um leve aumento. Os caldos de cocção também apresentaram relevante teor de ácido ascórbico, valores estes próximos aos do encontrado no grão cozido apresentando diferença estatística entre si para a maioria das cultivares.

O ácido ascórbico é um antioxidante natural que exerce grande importância ao metabolismo vegetal por estar envolvido na embriogênese, durante o processo de formação das sementes (Raza et al., 2013). É também uma das mais importantes vitaminas, sendo um indicador de sensibilidade dos alimentos por isso, é frequentemente usada como parâmetro de severidade no processamento. A destruição de vitaminas provocadas pelo tratamento térmico depende do tempo de exposição ao calor e do método de cocção utilizado. Portanto, a vitamina C por ser hidrossolúvel, pode ser retida pela água, tanto no método por imersão quanto nos métodos que utilizaram água sob pressão (Pigoli et al., 2014). Demonstrando o efeito do método utilizado no cozimento dos grãos em panela de pressão durante 10 minutos e o processo de lixiviação da vitamina $\mathrm{C}$ para o caldo de cocção.

Quadro 1. Conteúdo de ácido ascórbico em oito cultivares de feijão-caupi imaturo cru, cozido e caldo de cocção.

\begin{tabular}{|c|c|c|c|}
\hline \multirow{2}{*}{ Cultivares } & \multicolumn{3}{|c|}{ Ácido Ascórbico (mg.100g $\left.{ }^{-1}\right)$} \\
\cline { 2 - 4 } & Grão Cru & Grão Cozido & Caldo \\
\hline Costela de Vaca & $8,29^{\mathrm{a}}$ & $5,57^{\mathrm{a}}$ & $5,13^{\mathrm{a}}$ \\
\hline BRS Marataoã & $7,27^{\mathrm{ab}}$ & $5,57^{\mathrm{a}}$ & $4,01^{\mathrm{ab}}$ \\
\hline BRS Itaim & $4,15^{\mathrm{d}}$ & $4,23^{\mathrm{b}}$ & $3,34^{\mathrm{b}}$ \\
\hline BR 17-Gurgueia & $5,40^{\mathrm{cd}}$ & $5,12^{\mathrm{ab}}$ & $4,01^{\mathrm{ab}}$ \\
\hline BRS Novaera & $6,23^{\mathrm{bc}}$ & $6,02^{\mathrm{a}}$ & $5,35^{\mathrm{a}}$ \\
\hline Paulistinha & $6,85^{\mathrm{abc}}$ & $5,79^{\mathrm{a}}$ & $5,13^{\mathrm{a}}$ \\
\hline Setentão & $7,05^{\mathrm{ab}}$ & $5,79^{\mathrm{a}}$ & $5,13^{\mathrm{a}}$ \\
\hline Patativa & $5,39^{\mathrm{cd}}$ & $5,56^{\mathrm{a}}$ & $4,91^{\mathrm{ab}}$ \\
\hline
\end{tabular}

*Média de quatro repetições. Letras minúsculas iguais não apresentam diferença significativa entre as médias $(\mathrm{p}<0,05)$.

Fonte: Autores (2021). 
No Quadro 2 observa-se que nos grãos crus, o maior teor de clorofila foi registrado para cultivar Costela de Vaca com média de 7,23 mg. $100 \mathrm{~g}^{-1}$, que não diferiu significativamente da cultivar Setentão com $6,78 \mathrm{mg} .100 \mathrm{~g}^{-1}$ e menor média para a BR 17-Gurgueia com 2,07 mg.100g-1. Após o cozimento todas as cultivares apresentaram redução no teor de clorofila, com menores concentrações para Patativa $\left(0,48 \mathrm{mg} \cdot 100 \mathrm{~g}^{-1}\right)$ e Setentão $\left(0,62 \mathrm{mg} \cdot 100 \mathrm{~g}^{-1}\right)$, não diferindo estatisticamente entre si, e maiores concentrações nas cultivares BRS Marataoã (2,12 mg.100g $\left.{ }^{-1}\right)$ e Costela de Vaca $\left(2,35\right.$ mg. $\left.100 g^{-1}\right)$.

Quadro 2. Conteúdo de clorofila e carotenoides em oito cultivares de feijão-caupi imaturo cru e cozido.

\begin{tabular}{|c|c|c|c|c|}
\hline \multirow{2}{*}{ Cultivares } & \multicolumn{2}{|c|}{$\begin{array}{c}\text { Clorofila } \\
\left(\mathbf{m g . 1 0 0 g}^{-1}\right)\end{array}$} & \multicolumn{2}{c|}{ Carotenoides $\left.^{*} \mathbf{\mu g . 1 0 0 g}^{-1}\right)$} \\
\cline { 2 - 5 } & Grão Cru & Grão Cozido & Grão Cru & Grão Cozido \\
\hline Costela de Vaca & $7,23^{\mathrm{a}}$ & $2,35^{\mathrm{a}}$ & $3,04^{\mathrm{a}}$ & $1,03^{\mathrm{a}}$ \\
\hline BRS Marataoã & $4,70^{\mathrm{b}}$ & $2,12^{\mathrm{a}}$ & $2,08^{\mathrm{bc}}$ & $0,98^{\mathrm{a}}$ \\
\hline BRS Itaim & $4,40^{\mathrm{bc}}$ & $1,71^{\mathrm{ab}}$ & $1,72^{\mathrm{cd}}$ & $0,74^{\mathrm{abc}}$ \\
\hline BR 17-Gurgueia & $2,07^{\mathrm{d}}$ & $1,72^{\mathrm{ab}}$ & $1,06^{\mathrm{e}}$ & $0,77^{\mathrm{abc}}$ \\
\hline BRS Novaera & $3,23^{\mathrm{cd}}$ & $2,51^{\mathrm{a}}$ & $1,29^{\mathrm{e}}$ & $0,94^{\mathrm{ab}}$ \\
\hline Paulistinha & $3,22^{\mathrm{cd}}$ & $1,23^{\mathrm{ab}}$ & $1,28^{\mathrm{e}}$ & $0,65^{\mathrm{abc}}$ \\
\hline Setentão & $6,78^{\mathrm{a}}$ & $0,62^{\mathrm{b}}$ & $2,42^{\mathrm{b}}$ & $0,43^{\mathrm{bc}}$ \\
\hline Patativa & $3,41^{\mathrm{cd}}$ & $0,48^{\mathrm{b}}$ & $1,33^{\mathrm{de}}$ & $0,38^{\mathrm{c}}$ \\
\hline
\end{tabular}

*Média de quatro repetições. Letras minúsculas iguais não apresentam diferença significativa entre as médias $(\mathrm{p}<0,05)$.

Fonte: Autores (2021).

Oliveira et al, (2013), trabalhando com três cultivares de feijão caupi imaturo obteve médias que variaram de 3,00 mg.100g $\mathrm{g}^{-1}$ para a cultivar BRS-Guariba a 3,26 mg/100g para a cultivar BRS-Tumucumaque. A perda de clorofila causa mudança de cor nos vegetais, o que muitas vezes está associado com a perda de qualidade destes, quando utilizados como alimentos. Pela degradação da clorofila, a cor passa de verde-brilhante para verde-oliva em alimentos processados e uma ampla variedade de cores em tecidos senescentes, no caso de vegetais consumidos in natura. A clorofila é sensível ao pH, enzimas, temperatura, luz e oxigênio, os quais têm maior ou menor influência na sua degradação conforme a atividade de água do meio. Em virtude disso, a constituição do alimento e as condições de processamento, bem como o ambiente de armazenagem, influenciam grandemente no curso da degradação da clorofila em alimentos processados (Aquino et al., 2011).

Com relação ao teor de carotenoides pode-se observar no Quadro 2 que a cultivar Costela de Vaca com 3,04 $\mu \mathrm{g} / 100 \mathrm{~g}$, foi estatisticamente superior às demais cultivares para os grãos crus e cozidos. As menores médias foram observadas na cultivar BRS 17-Gurgueia $(1,06 \mu \mathrm{g} / 100 \mathrm{~g})$ no feijão-cru e no cozido a menor média foi obtida para a cultivar Patativa com $(0,38 \mu \mathrm{g} / 100 \mathrm{~g})$. Estes valores estão próximos aos encontrados por Umeda 2017, onde as duas variedades de feijão estudadas obtiveram médias de $0,16 \mathrm{mg} / 100 \mathrm{~g}$ (Carioca cru) e 0,41 mg/100g (Preto cru), não sendo detectados concentrações nos tratamentos com cocção.

Rodriguez-Amaya (2001) afirma que, fatores como luz, calor, ação enzimática e oxidação oriunda por peróxidos promovem a deterioração desses carotenoides e sua estabilidade pode diferir para cada alimento quando submetidos a processamentos, mas a principal causa da deterioração está ligada principalmente à oxidação, assim como também essa variação pode ser conforme a origem, devido à localização, clima, condições ambientais e categoria de solo onde são cultivados. 
Os resultados apresentados no Quadro 3 demonstram valores de flavonoides obtidos nas cultivares de feijão-caupi encontram-se no Quadro 2. As maiores concentrações de flavonoides foram apresentadas pelas cultivares Setentão e BR 17Gurgueia com 7,28 mg. $100 \mathrm{~g}^{-1}$ e $6,96 \mathrm{mg} 100 \mathrm{~g}^{-1}$ respectivamente, não diferindo estatisticamente entre si quando avaliadas na forma crua. O cozimento provocou uma leve redução no teor de flavonoides apenas para as cultivares Costela de Vaca (5,29 mg. $\left.100 \mathrm{~g}^{-1}\right)$ e Setentão $\left(6,60 \mathrm{mg} .100 \mathrm{~g}^{-1}\right)$, com aumento para as demais. Para os caldos de cocção, houve uma leve lixiviação de flavonoides dos grãos, com destaque de maior concentração para cultivar Costela de 0,29 (mg/100g).

Quadro 3. Conteúdo de flavonoides em oito cultivares de feijão-caupi imaturo cru, cozido e caldo de cocção.

\begin{tabular}{|c|c|c|c|}
\hline \multirow[t]{2}{*}{ Cultivares } & \multicolumn{3}{|c|}{$\begin{array}{c}\text { Flavonoides } \\
\left(\mathbf{m g . 1 0 0 g}^{-1}\right)\end{array}$} \\
\hline & Grão Cru & Grão Cozido & Caldo \\
\hline Costela de Vaca & $5,37^{\mathrm{ab}}$ & $5,29^{b}$ & $0,29^{\mathrm{a}}$ \\
\hline BRS Marataoã & $4,83^{\mathrm{ab}}$ & $5,39^{\mathrm{b}}$ & $0,18^{\mathrm{bc}}$ \\
\hline BRS Itaim & $4,13^{b}$ & $8,01^{\mathrm{ab}}$ & $0,14^{\mathrm{c}}$ \\
\hline BR 17-Gurgueia & $6,96^{\mathrm{a}}$ & $8,28^{\mathrm{a}}$ & $0,18^{\mathrm{bc}}$ \\
\hline BRS Novaera & $4,56^{\mathrm{ab}}$ & $6,00^{\mathrm{ab}}$ & $0,15^{\mathrm{c}}$ \\
\hline Paulistinha & $5,24^{\mathrm{ab}}$ & $6,34^{\mathrm{ab}}$ & $0,22^{\mathrm{b}}$ \\
\hline Setentão & $7,28^{\mathrm{a}}$ & $6,60^{\mathrm{ab}}$ & $0,22^{\mathrm{b}}$ \\
\hline Patativa & $5,18^{\mathrm{ab}}$ & $6,07^{\mathrm{ab}}$ & $0,16^{\mathrm{c}}$ \\
\hline
\end{tabular}

*Média de quatro repetições. Letras minúsculas iguais não apresentam diferença significativa entre as médias $(\mathrm{p}<0,05)$.

Fonte: Autores (2021).

Pinheiro (2013), avaliando três genótipos de feijão-caupi cru e cozido encontrou concentrações variando de 6,43

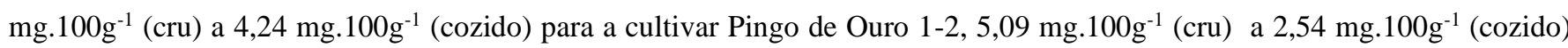
para a linhagem MMCO3-737F-5-9 e 3,90 mg.100g-1 (cru) a 2,71 mg.100g-1 (cozido) para a linhagem MNCO3-737F-5-4. Estes resultados assemelham-se aos do presente trabalho para o feijão-cru. Sendo que para o feijão cozido o referido autor observou uma redução nas concentrações de flavonoides para todos os genótipos avaliados, resultados estes contrários ao do presente trabalho no qual houve um aumento após o cozimento para seis das oito cultivares.

No Quadro 4 estão apresentados os valores de antocianinas obtidos nas cultivares de feijão-caupi. O conteúdo total de antocianinas nas cultivares estudadas variou de $1,07 \mathrm{mg} .100 \mathrm{~g}^{-1}$ (BRS Itaim - grão cru) a 0,04 mg.100g-1 (BRS Novaera - grão cozido). Essas baixas concentrações podem evidenciar diferentes efeitos do processamento e das cultivares em relação às antocianinas, o que pode ser atribuído à instabilidade destas em altas temperaturas, visto que são rapidamente destruídas pelo aquecimento. Barros et al., (2017) e Moreira-Araújo et al., (2018), não identificaram a presença de antocianinas em cultivares de feijão-caupi (BRS Xiquexique, BRS Milênio, BRS Aracê e BRS Tumucumaque), antes e depois do seu cozimento. Pesquisas realizadas com o intuito de identificar e quantificar antocianinas, flavonoides e taninos condensados em amostras de feijão-caupi com o tegumento escuro, apresentaram maiores concentrações, visto que esses compostos bioativos se concentram nesta região dos grãos (Ojwang et al., 2013). Nosso estudo confirma as declarações de Akond et al. (2011) sobre o relacionamento direto entre a cor do tegumento do feijão e o níveis de antocianinas, como a cultivar BRS Itaim possui um tegumento escuro foi então detectável a presença de maior concentração de antocianina nessa cultivar. 
Quadro 4. Conteúdo de antocianinas em oito cultivares de feijão-caupi imaturo cru, cozido e caldo de cocção.

\begin{tabular}{|c|c|c|c|}
\hline \multirow{2}{*}{ Cultivares } & \multicolumn{3}{|c|}{$\begin{array}{c}\text { Antocianinas } \\
\left(\mathbf{m g . 1 0 0}^{-1}\right)\end{array}$} \\
\cline { 2 - 4 } & Grão Cru & Grão Cozido & Caldo \\
\hline Costela de Vaca & $0,74^{\mathrm{abc}}$ & $0,22^{\mathrm{ab}}$ & $0,08^{\mathrm{b}}$ \\
\hline BRS Marataoã & $0,45^{\mathrm{bc}}$ & $0,11^{\mathrm{ab}}$ & $0,05^{\mathrm{c}}$ \\
\hline BRS Itaim & $1,07^{\mathrm{a}}$ & $0,35^{\mathrm{a}}$ & $0,10^{\mathrm{a}}$ \\
\hline BR 17-Gurgueia & $0,40^{\mathrm{c}}$ & $0,09^{\mathrm{ab}}$ & $0,05^{\mathrm{c}}$ \\
\hline BRS Novaera & $0,46^{\mathrm{bc}}$ & $0,04^{\mathrm{b}}$ & $0,05^{\mathrm{c}}$ \\
\hline Paulistinha & $0,49^{\mathrm{bc}}$ & $0,17^{\mathrm{ab}}$ & $0,05^{\mathrm{c}}$ \\
\hline Setentão & $0,79^{\mathrm{ab}}$ & $0,28^{\mathrm{ab}}$ & $0,06^{\mathrm{c}}$ \\
\hline Patativa & $0,58^{\mathrm{bc}}$ & $0,34^{\mathrm{a}}$ & $0,05^{\mathrm{c}}$ \\
\hline
\end{tabular}

*Média de quatro repetições. Letras minúsculas iguais não apresentam diferença significativa entre as médias $(\mathrm{p}<0,05)$.

Fonte: Autores (2021).

O Quadro 5 mostra o conteúdo de compostos fenólicos totais obtidos nas oito cultivares de feijão-caupi verde, cru, cozido e caldo de cocção. Pode-se observar que para o grão cru, as oito cultivares apresentaram diferença estatística entre si, com maior concentração para cultivar BRS Marataoã com $87,74 \mathrm{mg} \cdot 100 \mathrm{~g}^{-1}$, seguida da cultivar Paulistinha com 81,58 mg. $100 \mathrm{~g}^{-1}$. As cultivares BR 17-Gurgueia e BRS Novaera foram as que apresentaram os menores teores, com 44,19 e 45,00 mg. $100 \mathrm{~g}^{-1}$, respectivamente.

Com relação aos grãos cozidos, todos diferiram estatisticamente entre si $(\mathrm{p}<0,05)$, com a cultivar Setentão apresentando a maior concentração 46,29 mg.100g $\mathrm{g}^{-1}$, e as cultivares BRS Itaim $\left(26,08 \mathrm{mg}_{1} 100 \mathrm{~g}^{-1}\right)$ e BRS Novaera $(26,05$ $\left.\mathrm{mg} .100 \mathrm{~g}^{-1}\right)$ com as menores concentrações. Em todas as cultivares, foi observada uma diminuição na concentração de fenólicos após o cozimento, constatando-se que o processamento reduz consideravelmente o teor desses compostos, provavelmente devido às alterações nas moléculas ocasionadas pelo aumento da temperatura, transformando-os em outras substâncias.

Todos os caldos, apresentaram concentrações de fenólicos, com a maior proporção para cultivar BRS Marataoã (51,88 mg. $100 \mathrm{~g}^{-1}$ ), observou-se também que a cultivar Costela de Vaca obteve uma maior concentração de compostos fenólicos no caldo de cocção $\left(43,34 \mathrm{mg} \cdot 100 \mathrm{~g}^{-1}\right)$ em comparação ao grão cozido $\left(30,62 \mathrm{mg} \cdot 100 \mathrm{~g}^{-1}\right)$. 
Quadro 5. Conteúdo de compostos fenólicos em oito cultivares de feijão-caupi imaturo cru, cozido e caldo de cocção.

\begin{tabular}{|c|c|c|c|}
\hline \multirow[t]{2}{*}{ Cultivares } & \multicolumn{3}{|c|}{$\begin{array}{c}\text { Compostos Fenólicos } \\
\left(\mathbf{m g . 1 0 0 g}^{-1}\right)\end{array}$} \\
\hline & Grão Cru & Grão Cozido & Caldo \\
\hline Costela de Vaca & $70,48^{\mathrm{bc}}$ & $30,62^{b c}$ & $43,34^{\mathrm{b}}$ \\
\hline BRS Marataoã & $87,74^{\mathrm{a}}$ & $28,49^{\mathrm{bc}}$ & $51,88^{\mathrm{a}}$ \\
\hline BRS Itaim & $61,69^{c}$ & $26,08^{\mathrm{c}}$ & $20,08^{f}$ \\
\hline BR 17-Gurgueia & $44,19^{\mathrm{d}}$ & $33,70^{\mathrm{b}}$ & $18,86^{\mathrm{fg}}$ \\
\hline BRS Novaera & $45,00^{\mathrm{d}}$ & $26,05^{\mathrm{c}}$ & $15,64^{\mathrm{g}}$ \\
\hline Paulistinha & $81,58^{a b}$ & $32,30^{\mathrm{b}}$ & $31,50^{\mathrm{d}}$ \\
\hline Setentão & $79,39^{\mathrm{ab}}$ & $46,29^{a}$ & $37,31^{\mathrm{c}}$ \\
\hline Patativa & $79,44^{\mathrm{ab}}$ & $33,39^{\mathrm{b}}$ & $26,43^{\mathrm{e}}$ \\
\hline
\end{tabular}

*Média de quatro repetições. Letras minúsculas iguais não apresentam diferença significativa entre as médias $(\mathrm{p}<0,05)$.

Fonte: Autores (2021).

Tem-se relatado constantemente na literatura a contribuição do feijão-caupi no aporte de compostos fenólicos para a população. Em pesquisas de Barros et al. (2017); Cavalcante et al. (2017) foi observado uma variação de 89,43 a 295,23 mg.100g ${ }^{-1}$ em grãos de nove cultivares de feijão-caupi. Moreira-Araújo et al. (2018) identificaram entre os compostos fenólicos contidos nos grãos de feijão-caupi, o ácido gálico $\left(45,4\right.$ e 93,4 mg.100g $\left.\mathrm{g}^{-1}\right)$, catequina $(5,57$ e 6,48 mg.100g-1), epicatequina $(8,67$ e 2,95 mg.100g-1 $)$, ácido ferúlico $\left(11,1\right.$ e $\left.13,8 \mathrm{mg} .100 \mathrm{~g}^{-1}\right)$ e ácido clorogênico $\left(2,39\right.$ e $\left.0,59 \mathrm{mg} .100 \mathrm{~g}^{-1}\right)$ nos grãos da cultivar BRS Tumucumaque e da linhagem Pingo de Ouro 1-2, respectivamente.

Segundo Moreira-Araújo et al., (2018), vários fatores podem interferir no conteúdo de compostos fenólicos em legumes, como genéticos e ambientais, além de fatores inerentes às condições de extração destes compostos da matriz alimentar, como a categoria de solvente utilizado, por exemplo. Dessa forma, isso pode justificar as diferenças observadas no conteúdo destes compostos quando comparados com outros estudos. Segundo os autores apesar da redução dos compostos fenólicos após o cozimento, os teores desses compostos continuam expressivos, considerando-se o somatório dos conteúdos obtidos nas cultivares cozidas e respectivos caldos de cocção, mostrando que mesmo após o cozimento, as cultivares analisadas ainda constituem importantes aliados à saúde com preservação da sua funcionalidade.

O método para determinação da atividade antioxidante, utilizado neste trabalho, consiste na redução do radical DPPH (coloração púrpura) que, ao receber um elétron ou um radical hidrogênio, altera sua coloração para amarelo, ficando estável. O desaparecimento da coloração pode ser avaliado pelo decréscimo da absorbância. Quanto menor o valor de EC $_{50}$ (concentração de extrato capaz de reagir com 50\% do radical presente na solução de DPPH) maior é sua atividade antioxidante, pois será necessária uma menor quantidade de extrato para reduzir 50\% do radical livre DPPH.

Através dos resultados obtidos na análise de DPPH pelo EC50 (g feijão.g de $\mathrm{DPPH}^{-1}$ ), onde valores menores indicam maior atividade antioxidante, observa-se que houve diferença estatisticamente significativa entre os feijões-crus. A cultivar BRS Marataoã foi a que apresentou a menor concentração $\left(97,71\right.$ g feijão.g DPPH $\left.{ }^{-1}\right)$ e consequentemente a maior capacidade em reduzir em 50\% o radical DPPH. Comportamento diferente foi observado nos grãos cozidos, com a cultivar Setentão apresentando a menor concentração (129,32 g feijão.g DPPH $\left.{ }^{-1}\right)$ obtendo o melhor desempenho. Nos caldos de cocção, as cultivares BRS Marataoã e Costela de Vaca foram as que apresentaram as menores concentrações e consequentemente maior capacidade em combater o radical livre. Após o cozimento, constatou-se uma diminuição estatisticamente significativa na 
atividade antioxidante das cultivares, com exceção da BR 17-Gurgueia que obteve maior atividade antioxidante no grão cozido com 147,76 g feijão.g DPPH-1 que no grão cru com 179,31 g feijão.g DPPH ${ }^{-1}$.

Quadro 6. Capacidade antioxidante de oito cultivares de feijão-caupi imaturo cru, cozido e caldo de cocção.

\begin{tabular}{|c|c|c|c|}
\hline \multirow{2}{*}{ Cultivares } & \multicolumn{3}{|c|}{$\begin{array}{c}\text { Capacidade Antioxidante EC } \\
\text { (g0 } \\
\text { (g feijão.g DPPH }\end{array}$} \\
\cline { 2 - 4 } & Grão Cru & Grão Cozido & Caldo \\
\hline Costela de Vaca & $119,04^{\mathrm{b}}$ & $194,52^{\text {ab }}$ & $83,60^{\text {cd }}$ \\
\hline BRS Marataõa & $97,71^{\mathrm{c}}$ & $218,71^{\mathrm{ab}}$ & $79,59^{\mathrm{d}}$ \\
\hline BRS Itaim & $165,94^{\mathrm{a}}$ & $239,56^{\mathrm{ab}}$ & $172,78^{\mathrm{b}}$ \\
\hline BR 17-Gurgueia & $179,31^{\mathrm{a}}$ & $147,76^{\mathrm{ab}}$ & $245,08^{\mathrm{a}}$ \\
\hline BRS Novaera & $178,68^{\mathrm{a}}$ & $276,11^{\mathrm{a}}$ & $254,40^{\mathrm{a}}$ \\
\hline Paulistinha & $103,66^{\mathrm{c}}$ & $163,53^{\mathrm{ab}}$ & $31,50^{\mathrm{d}}$ \\
\hline Setentão & $109,72^{\mathrm{bc}}$ & $129,32^{\mathrm{b}}$ & $37,31^{\mathrm{c}}$ \\
\hline Patativa & $111,19^{\mathrm{bc}}$ & $147,92^{\mathrm{ab}}$ & $26,43^{\mathrm{e}}$ \\
\hline
\end{tabular}

*Média de quatro repetições. Letras minúsculas iguais não apresentam diferença significativa entre as médias $(\mathrm{p}<0,05)$.

Fonte: Autores (2021).

Dentre os compostos bioativos analisados, houve uma forte correlação entre os compostos fenólicos, flavonoides totais e ácido ascórbico dos extratos dos feijões e a atividade antioxidante dos mesmos, visto que a cultivar BRS Marataoã crua, a cultivar Setentão cozida e os caldos de cocção das cultivares BRS Marataoã e Costela de Vaca apresentaram os maiores teores de compostos fenólicos, que se refletiu nas suas maiores capacidades antioxidantes. De acordo com Barros, (2014), a redução da ação antioxidante após o cozimento pode ocorrer devido ao processamento térmico promover a destruição de compostos bioativos levando a redução dos mesmos, e/ou à formação de novos compostos com ação pró-oxidante, o que foi observado no presente trabalho, com exceção da cultivar BR 17-Gurgueia que obteve maior atividade antioxidante no cozido $147,76 \mathrm{~g}$ feijão.g DPPH ${ }^{-1}$ que no feijão-cru com 179,31 g feijão.g DPPH ${ }^{-1}$.

\section{Considerações Finais}

A presença de clorofila, carotenoides e antocianinas nas cultivares foi detectada em pequenas concentrações após o cozimento. O conteúdo de flavonoides apresentou um aumento após o cozimento em seis das oito cultivares analisadas. O teor de ácido ascórbico diminuiu após o cozimento para todas as cultivares com exceção da cultivar Patativa. O cozimento também afetou o conteúdo de compostos fenólicos, porém, houve um aumento deste, nos caldos de cocção para as cultivares Costela de Vaca e BRS Marataoã.

Para a atividade antioxidante, antes do cozimento, a cultivar BRS Marataoã apresentou maior atividade antioxidante. Após o cozimento, o caldo de cocção apresentou melhores resultados em relação aos grãos cozidos para seis das oito cultivares analisadas. Os compostos fenólicos totais, os flavonoides e o ácido ascórbico foram os principais compostos bioativos a contribuir para a atividade antioxidante das cultivares de feijão-caupi imaturo.

Após o processamento, as cultivares mantiveram suas características nutritivas e funcionais consideráveis, podendo recomendar o consumo do feijão-caupi imaturo com o seu caldo de cocção para melhor aproveitamento dos seus compostos com propriedades antioxidantes. 
Em trabalhos futuros, recomendamos estudos que avaliem outras cultivares de feijão-caupi imaturo, aumentando assim o conhecimento sobre a potencialidade funcional dessa cultura assim como a identificação desses compostos bioativos.

\section{Referências}

Aquino, A. C. M. S., Silva, M. H. M., Rocha, A. K. S. \& Castro, A. A. (2011). Estudo da influencia de diferentes tempos e métodos de cocção na estabilidade dos teores de clorofila e ácido ascórbico em brócolis (Brassica oleraceae). Scientia Plena. 7(1), 1-6. https://www.scientiaplena.org.br/sp/article/view/172

Akond, G. M., Crawford, H., Berthold, J., Talukder, Z. I. \& Hossain K. (2011). Minerals (Zn, Fe, Ca and Mg) and antinutrient (phytic acid) constituents in common bean. American Journal of Food Technology, 6 (3), 235-243. 10.3923/ajft.2011.235.243

Andrade, F.N., Rocha, M.M., Gomes, R.L.F., Freire Filho, F.R., Silva, K. J. D., Rodrigues, E.V. \& Silva, L.R.A. (2011). Potencial nutricional e culinário de linhagens de tegumento e cotilédone verdes para o mercado de feijão-caupi verde. IV Reunião de Biofortificação.

Association of Official Analytical Chemists. (2005). Official Methods of Analysis of AOAC International. (18a ed.). Gaithersburg, Maryland.

Barros, N. V. A. (2014). Influência do cozimento na composição centesimal, minerais, compostos bioativos e atividade antioxidante de cultivares de feijãocaupi. 2014. 90 f. Dissertação (Mestrado) - Programa de Pós-Graduação em Alimentos e Nutrição, Universidade Federal do Piauí, Teresina-PI.

Barros, N. V. dos A., Rocha, M. de M., Glória, M. B. A, Araújo, M. A. da M. \& Moreira-Araújo, R. S. dos R. (2017). Effect of cooking on the bioactive compounds and antioxidant activity in grains cowpea cultivars. Revista Ciência Agronômica, Fortaleza. 48 (5), 824-831. 10.5935/1806-6690.20170097

Cavalcante, R. B. M., Araújo, M. A. M., Rocha, M. M. \&Moreira-Araújo, R. S. R. (2017). Effect of thermal processing on chemical compositions, bioactive compounds, and antioxidant activities of cowpea cultivars. Revista Caatinga. 30 (4), 1050-1058. 10.1590/1983-21252017v30n426rc

Francis, F.J. (1982). Analysis of anthocyanins: Anthocyanins as food colors. Academic Press, 181-207.

Freire Filho, F. R., Ribeiro, V. Q., Rocha, M. de M., Silva, K. J. D., Nogueira, M. do S. da R. \& Rodrigues, E. V. (2011). Feijão-caupi: produção, melhoramento genético, avanços e desafios. Brasília, Embrapa Informação Tecnológica. 81p, 2011.

Ial, I. A. L. (2008). Métodos físico-químicos para análise de alimentos. IAL, Normas Analíticas.

Larrauri, J. A., Rupérez, P., Saura-Calixto, F. (1997). Effect of drying temperature on the stability of polyphenols and antioxidant activity of red grape pomece peels. Journal of Agricultural and Food Chemistry. 45 (4), 1390-1393. https://pubs.acs.org/doi/abs/10.1021/jf960282f

Lichtenthaler, H. A. (1987). Chlorophylls and carotenoids: pigments of photosynthetic membranes. Methods Enzymol. 148, $350-383$.

Locatelli, V. da E. R., Medeiros, R. D. de, Smiderle, O. J., Albuquerque, J. de A. A., Araújo, W. F. \& Souza, K. T. S. de. (2014). Componentes de Produção, produtividade e eficiência da irrigação do feijão-caupi no cerrado de Roraima. Revista Brasileira de Engenharia Agrícola e Ambiental. 18 (6), 574-580. 10.1590/S1415-43662014000600002

Manaf, H. H., Zayed, M. S. (2015). Productivity of cowpea as affected by salt stress in presence of endomycorrhizae and Pseudomonas fluorescens. Annals of Agricultural Science. 60 (2), 219-226. 10.1016/j.aoas.2015.10.013

Moreira-Araújo, R.S.R., Sampaio, G. R., Soares, R. A. M., Silva, C. P., Araújo, M. A. M. \& Arêas, J. A. G. (2018). Identification and quantification of phenolic compounds and antioxidant activity in cowpeas of BRS Xiquexique cultivar. Revista Caatinga. 31 (1), 209-216. 10.1590/1983-21252018v31n124rc

Nderitu, A. M., Dykes, L., Awika, J. M., Minnaar, A. \& Duodu, K. G. (2013). Phenolic composition and inhibitory effect against oxidative DNA damage of cooked cowpeas as affected by simulated in vitro gastrointestinal digestion. Food Chemistry, Barking. 141 (3), 1763-1771. 10.1016/j.foodchem.2013.05.001

Ojwang, L. O., Dykes, L. \& Awika, J. M. (2013). Proanthocyanidin profile of cowpea (Vigna unguiculata) reveals catechin-O-glucoside as the dominant compound. Food Chemistry, Barking. 139 (1-4), 35-43..1016/j.foodchem.2013.01.117

Oliveira, V. R., Ribeiro, N. D., Jost, E., Colpo, E. \& Poersch, N. L. (2013). Perfil sensorial de cultivares de feijão sob diferentes tempos de cozimento. Alimentos e Nutrição, Araraquara. 24 (2), 145-152.

Pereira, T. S., Lima, M. D. R., Paula, L. S. \& Lobato, A. K. S. (2016). Tolerance to water deficit in cowpea populations resulting from breeding program: detection by gas exchange and chlorophyll fluorescence. Indian Journal of Plant Physiology. 21(2), 171-178. 10.1007/s40502-016-0218-3

Pigoli, D. R., Vieites, R. L. \& Daiuto, E. R. (2014). Alterações nutricionais em casca e polpa de cenoura decorrente de diferentes métodos de cozimento. Revista Energia na Agricultura, Botucatu. 29 (2), 121-127. 10.17224/EnergAgric.2014v29n2p121-127

Pinheiro, E. M. (2013). Caracterização Química, poder antioxidante e efeito do cozimento de genótipos de feijão-caupi. Dissertação (Mestrado em Alimentos e Nutrição) - Universidade Federal do Piauí, Piauí.

Raza, S. H., Shafiq, F., Chaudhary M. \& Khan, I. (2013). Seed invigoration with water, ascorbic and salicylic acid stimulates development and biochemical characters of okra (Ablemoschus esculentus) under normal and saline conditions. International Journal of Agriculture and Biology 15 (3), $486-492$.

Rodriguez-Amaya, D. B. A Guide to Carotenoid Analysis in Foods.

Rufino, M. S. M., Alves, R. E., Brito, E. S., Morais, S. M., Sampaio, C. G., Pérez-Jiménez, J. \& Saura-Calixto, F. D. (2007). Metodologia científica: determinação da atividade antioxidante total em frutas pela captura do radical livre DPPH. Comunicado Técnico. Empresa Brasileira de Pesquisa Agropecuária. 127, 4p. 
Research, Society and Development, v. 10, n. 7, e3710716243, 2021

(CC BY 4.0) | ISSN 2525-3409 | DOI: http://dx.doi.org/10.33448/rsd-v10i7.16243

Silva, E.F., Barros-Junior, A.P., Silveira, L.M. da, Santana, F.M. de S. \& Santos, M.G. dos. (2013). Avaliação de cultivares de feijão-caupi irrigado para produção de grãos verdes em Serra Talhada-PE. Revista Caatinga. 26 (1), 21-26. https://periodicos.ufersa.edu.br/index.php/caatinga/article/view/2650

Silva Junior, J. S. da. (2018). Desempenho produtivo do feijão-caupi sob diferentes densidades de plantas e regimes hídricos. 2018 . 69 f. Dissertação (Mestrado) - Universidade Federal do Piauí, Teresina.

Sombié, P. A. E. D., Compaoré, M., Coulibaly, A. Y., Ouédraogo, J. T., Tignégré, J. B. S. \& Kiendrébéogo, M. (2018). Antioxidant and Phytochemical Studies of 31 Cowpeas (Vigna unguiculata (L. Walp.)) Genotypes from Burkina Faso. Foods. 7(143), 1-9. 10.3390/foods7090143

Umeda, W. M. (2017). Caracterização nutricional, capacidade antioxidante e compostos bioativos de grãos de feijão (Phaseolus vulgaris L.). 2017. 100 f. Dissertação (Mestrado) - Universidade Estadual Paulista "Júlio de Mesquita Filho", Instituto, Letras e Ciências Exatas, São José do Rio Preto. https://repositorio.unesp.br/handle/11449/150161?show=full

Yenm, E. W., \& Willis, A. J. (1954). The estimation of carbohydrates in plant extracts by anthrone. Biochemical Journal. 57, 769-773.

Waterhouse, A. (2011). Folin-ciocalteau micro method for total phenol in wine. American Journal of Enology and Viticulture, p. 3-5. 